\title{
Nocebo hyperalgesia: contributions of social observation and body-related cognitive styles
}

This article was published in the following Dove Press journal:

Journal of Pain Research

22 April 2016

Number of times this article has been viewed

\author{
Elisabeth Vögtle' \\ Birgit Kröner-Herwig' \\ Antonia Barke ${ }^{2}$ \\ 'Department of Clinical Psychology \\ and Psychotherapy, Georg-Elias- \\ Müller-Institute for Psychology, \\ University of Göttingen, Göttingen, \\ 2Department of Psychology, \\ Division of Clinical Psychology and \\ Psychotherapy, Philipps University \\ Marburg, Marburg, Germany
}

Correspondence: Elisabeth Vögtle Department of Clinical Psychology and Psychotherapy, Georg-Elias-MüllerInstitute for Psychology, University of Göttingen, Goßlerstr I4,

D-37073 Göttingen, Germany

Tel +4955I 3920243

Fax +4955133544

Email evoegtl@psych.uni-goettingen.de
Purpose: Recently, it has been shown that nocebo hyperalgesia can be acquired through observational learning. The aim of this study was to investigate socially induced nocebo hyperalgesia and its relationship with pain catastrophizing, somatic complaints, hypochondriacal concerns, and empathy.

Participants and methods: Ninety-seven women (43.1 \pm 15.5 years) were randomly assigned to one of the two conditions. Participants in the nocebo condition (NC) watched a video in which a female model displayed more pain when an ointment was applied and less pain when no ointment was applied. In the control condition (CC), the model demonstrated low pain with and without the ointment. Subsequently, all participants received three pressure pain stimuli (60 seconds) on each hand. On one hand, the ointment was applied prior to the stimulation. The order of the stimulation of the fingers (middle, index, or ring finger), the side of ointment application (left or right hand), and the side with which the stimulation began were randomized within each group and balanced across the groups. Depending on the randomization, the pressure pain application started with or without ointment and on the left or right hand. Pain ratings on a numerical rating scale (0-10) were collected. In addition, the participants completed questionnaires regarding body-related cognitive styles and empathy.

Results: There was a significant difference in the pain ratings between the CC and the NC. The effect of ointment application was also significant, but no interaction between condition and ointment application was found. Only in the CC did the nocebo response correlate with hypochondriacal concerns and somatic complaints.

Conclusion: Application of an ointment as well as the observation of a model demonstrating more pain after a treatment produced elevated pain ratings. Cognitive styles were not related to the socially induced nocebo response, but were related to the nocebo response in the CC.

Keywords: nocebo response, pain, social observation, pain catastrophizing, hypochondriasis

\section{Introduction}

Expecting a negative outcome from an intervention may give rise to the very occurrence of that outcome. This phenomenon, called the "nocebo effect", can be induced by conditioning, verbal suggestion, ${ }^{1,2}$ or, as recently shown, by social observation; with student samples, two studies independently showed that observing a person who experienced pain after a particular intervention could lead to nocebo hyperalgesia when the observer underwent the same procedure. ${ }^{3,4}$ In one of the studies, four groups observed a model who reported lower pain ratings when a green rather than a red light preceded a pseudo-electric shock, whereas two other groups observed no model. ${ }^{3}$ Participants in all groups received electric shocks. The aim of the study was 
to investigate socially induced placebo analgesia with the green light-coupled shocks; however, when compared to the control groups without observation, a nocebo hyperalgesia to the red light-coupled electric shocks was induced. In the second study, female students watched a video model who rated pain as higher when an ointment was applied prior to the application of pressure pain. ${ }^{4}$ This also resulted in nocebo hyperalgesia in the observer.

Very little is known about why some people respond to placebos while others do not; ${ }^{5}$ the same is true for nocebos. For socially induced nocebo hyperalgesia, there was an effect of the model's sex; observing a male model led to a stronger hyperalgesia regardless of the observer's sex. ${ }^{3}$ Empathy might be a factor specifically related to socially induced nocebo hyperalgesia, as it might facilitate observational learning. However, results thus far are inconsistent. ${ }^{3}$ Studies concerning other factors influencing the nocebo response, as such, are rare. Personality variables, such as suggestibility, imaginative involvement, social desirability, and neuroticism, appear to be unrelated to verbally induced nocebo hyperalgesia. ${ }^{2}$ The cognitive-emotional variables pain anxiety and somatosensory amplification showed no correlation with nocebo hyperalgesia, whereas pain catastrophizing did show a correlation: the stronger the tendency to catastrophize, the stronger the nocebo hyperalgesia. ${ }^{4}$

In the present study, we further investigated cognitive processing styles that may be related to the nocebo response. The expectation of adverse somatosensory events, ${ }^{6}$ catastrophic misinterpretation, or overinterpretation of body sensations or physical symptoms ${ }^{7}$ and selective attention to bodily processes can affect the encoding of sensory information and impact symptom reports in general, ${ }^{8,9}$ and - we hypothesize - nocebo responses in particular. In addition, hypochondriacal concerns may influence the nocebo response, as they correlated positively with the number of retrospective reports of vicarious learning experiences related to bodily symptoms during childhood, and the authors concluded that persons with hypochondriacal concerns learned about symptoms through social observation. ${ }^{10}$

The aim of the present study was to investigate further socially induced nocebo effects and possible moderators of the socially induced nocebo hyperalgesia, such as pain catastrophizing, somatic complaints, hypochondriacal concerns, and empathy, in a sample from the general population.

In order to be terminologically unequivocal, we will distinguish between "nocebo effect" and "nocebo response". The term "nocebo effect" is reserved for statistically ascertained differences at the group level, whereas the term "nocebo response" refers to the difference resulting from subtracting the pain ratings without nocebo induction from the pain ratings with nocebo induction at an individual level.

\section{Participants and methods Ethics}

The study was approved by the local ethics committee (Ethikkommission des Instituts für Psychologie, GeorgAugust-Universität Göttingen, Approval number 55) and was carried out in accordance with the Declaration of Helsinki. ${ }^{11}$ Informed written consent was obtained prior to testing. Since a deceptive information procedure was used, after the experiment, the participants were fully debriefed regarding the deceptive information and the necessity of using this procedure, and were given the opportunity to ask questions.

\section{Participants}

Participants were recruited via newspaper and billboard advertisements. Differences in pain sensitivity between healthy men and women have been replicated in numerous studies ${ }^{12}$ which showed that experimenter sex influenced pain reports ${ }^{13,14}$ and that the sex of the model ${ }^{3}$ and participants ${ }^{15}$ influenced socially induced nocebo effects. Therefore, we kept these factors constant by employing a female model and including only women in our study. For the power analysis, we used the effect size of partial $\eta^{2}=0.09$ found for the interaction in our last paper as an approximation. We converted it via $\mathrm{G}^{*}$ Power to the effect size $f(\mathrm{~V})=0.31 .{ }^{16}$ A minimum total sample size of $\mathrm{N}=82$ participants was required to detect an interaction effect of that size $(\alpha=0.05$ and power $=0.80)$. Since, in our last study, participants had to be excluded, we decided to recruit more participants than the minimum required. A total of 109 healthy, right-handed women were randomly assigned to one of the two conditions. Twelve women were excluded after testing but before data analyses, as they reported suffering from depression (four), anorexia nervosa (one), anxiety disorders (two), agoraphobia (one), arthrosis (one), chronic back pain (one), fibromyalgia (one), and craniomandibular dysfunction (one). The remaining 97 participants (mean age nocebo condition [NC] 41.3 \pm 15.5 years, mean age control condition [CC] $44.7 \pm 15.5$ years) were included in the analysis. The groups did not differ with regard to age (Table 1).

\section{Design}

A $2 \times 2$ mixed design with the between-factor "condition" (nocebo/neutral) and within-factor "ointment application" (yes/no) was employed. All participants received pressure pain 
Table I Mean, standard deviation, and independent $t$-test results of participants' age, questionnaire scores, and credibility ratings for the nocebo and control conditions

\begin{tabular}{|c|c|c|c|c|c|c|}
\hline \multirow[t]{2}{*}{ Variable } & \multicolumn{2}{|c|}{$\begin{array}{l}\text { NC } \\
(n=47)\end{array}$} & \multicolumn{2}{|c|}{$\begin{array}{l}C C \\
(n=50)\end{array}$} & \multirow[t]{2}{*}{$t$} & \multirow[t]{2}{*}{$P$-value } \\
\hline & $\mathbf{M}$ & SD & $M$ & SD & & \\
\hline Age & 41.3 & 15.5 & 44.7 & 15.5 & 1.09 & 0.28 \\
\hline IRI & 40.7 & 5.2 & 41.4 & 4.0 & 0.76 & 0.45 \\
\hline PCS & 17.6 & 9.8 & 17.6 & 6.8 & 0.04 & 0.97 \\
\hline HADS-Dep & 4.5 & 3.5 & 3.6 & 3.1 & -1.23 & 0.22 \\
\hline HADS-Anx & 6.5 & 4.3 & 6.2 & 3.2 & -0.38 & $0.7 \mathrm{I}$ \\
\hline BL-R & 18.2 & 10.8 & 16.5 & 10.1 & -0.81 & 0.42 \\
\hline WI & 3.3 & 4.1 & 2.8 & 2.4 & -0.77 & 0.44 \\
\hline Credibility & 7.7 & 2.3 & 7.8 & 2.3 & 0.05 & 0.96 \\
\hline
\end{tabular}

Abbreviations: NC, nocebo condition; CC, control condition; IRI, Interpersonal Reactivity Index; PCS, Pain Catastrophizing Scale; HADS-Dep, Subscale Depression of the Hospital Anxiety and Depression Scale; HADS-Anx, Subscale Anxiety of the Hospital Anxiety and Depression Scale; BL-R, Symptom Inventory (Beschwerdeliste); WI, Whiteley Index; M, mean; SD, standard deviation.

stimuli for 60 seconds per finger on the middle phalanx of the ring, middle, and index finger of each hand. The dependent measure was subjective pain intensity. Assignment to the conditions was randomized. The order of stimulation of the fingers (middle phalanx of index, middle, and ring finger), the starting hand (right/left), the side of the ointment application (left/right), and whether the person's first or second hand was treated with ointment were balanced between the groups. Upon the participant's arrival at the laboratory, the experimenter drew a "lot" out of an envelope specifying these factors.

\section{Procedure}

Participants were contacted via the telephone and were sent a set of questionnaires (Symptom Inventory, Whiteley Index [WI], Hospital Anxiety and Depression Scale [HADS-D], see the "Measures" section) to fill out at home prior to coming to the laboratory. Participants received 20 euros for their participation.

Upon arrival at the laboratory, participants read the information that the influence of nonverbal instruction in pain experiments would be tested. To this end, they would be asked to watch a video in which the procedure was demonstrated by actions. No information concerning the possible effect of the ointment was provided. To focus their attention on the video, they received five questions about the video prior to viewing and were told that they would have to answer the questions after the video. The conditions differed solely with regard to the model's pain ratings in the video the participants were shown; in the NC, the model reported higher pain ratings after the application of an ointment, and in the $\mathrm{CC}$, the model's ratings were low throughout (see the "Video" section).
After they viewed the video and answered the related questions, the participants underwent the same procedure as they observed in the video. Depending on the randomization, pressure pain application started with or without ointment. The ointment (see the "Materials" section) was applied on the middle phalanx of the index, middle, and ring finger of one hand and allowed to take effect for 60 seconds as shown in the video. Pressure pain was applied by a stationary pressure pain algometer (see the "Materials" section) on the middle phalanx of the index, middle, and ring finger for 60 seconds on each of the three fingers of both hands. During the application, participants were asked to indicate the intensity of the pain verbally every 20 seconds on an eleven-point numerical rating scale with 0 indicating "no pain at all" and 10 indicating "the worst pain imaginable". The investigators recorded responses.

After the pain application procedure, participants filled out the remaining questionnaires (Interpersonal Reactivity Index [IRI] and Pain Catastrophizing Scale [PCS]). Finally, they wrote down which questions they thought were being examined by the study and rated the credibility of the cover story. An overview of the procedure is presented in Figure 1.

To increase the credibility of the procedure, professional surroundings for the experiment were created (health care

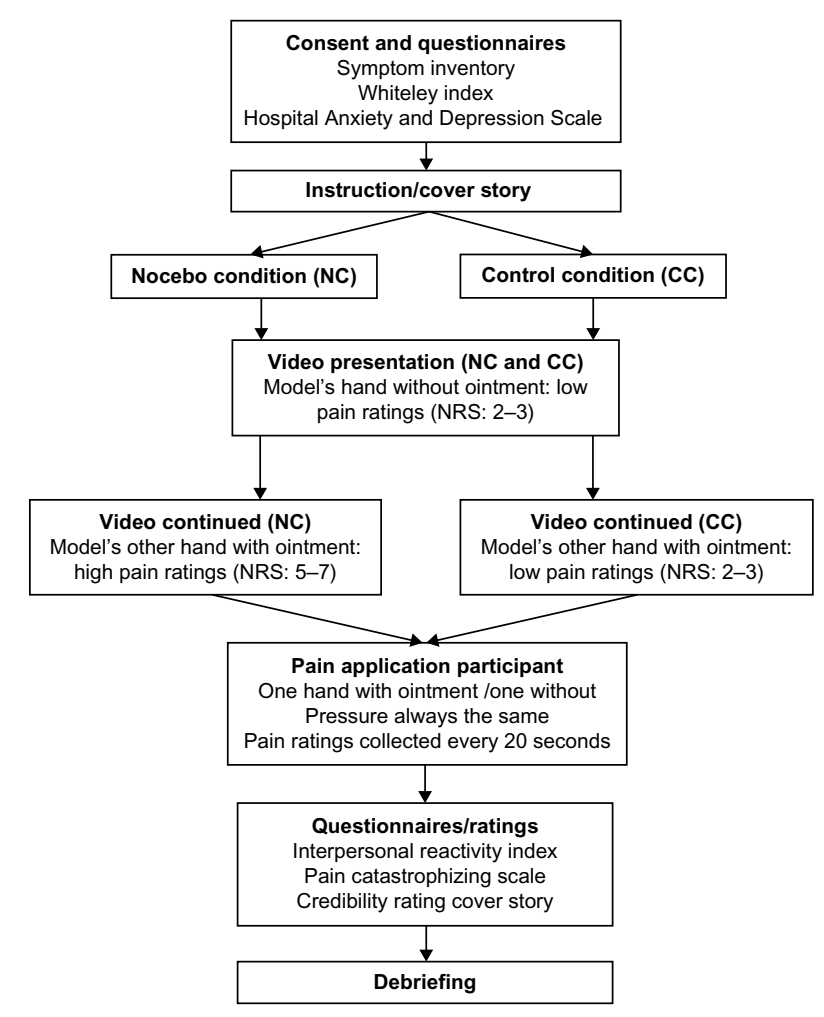

Figure I Experimental procedure.

Abbreviation: NRS, numerical rating scale. 
products, disinfectant spray), and the experimenters wore white medical coats and gloves when applying the ointment. The experimenters were trained and supervised by the first and senior authors (EV and AB). They were blind to the conditions and were only given code numbers of the videos to be shown. The participants wore headphones while watching the video, so that only they could hear the pain ratings. The group assignments were revealed to the experimenters only after the data from all participants had been collected.

\section{Video}

The videos were recorded in the same room in which the subsequent experiment took place. Both videos showed a seated female model in her mid-forties and the hands of a female experimenter. The videos were recorded from an angle behind the model so that the model's face was only partially visible (Figure 2). The videos lasted 10 minutes and 3 seconds each. In both videos, the experimenter told the model that pressure pain stimuli would be applied and explained the rating scale on which the pain intensity was to be rated. The pressure application started on the right hand without ointment. The model rated the pain intensity verbally on the eleven-point numerical rating scale. The model's ratings for the hand without ointment in both conditions ranged from 2 to 3. After all three fingers of the right hand had been stimulated, an ointment was applied to the model's left hand and allowed to take effect for 60 seconds. Then the application of pressure pain resumed. In the video for the NC, the model demonstrated an increase in pain after the ointment application by rating the pain for the hand with ointment from 5 to 7 . In the video for the $\mathrm{CC}$, the model demonstrated no

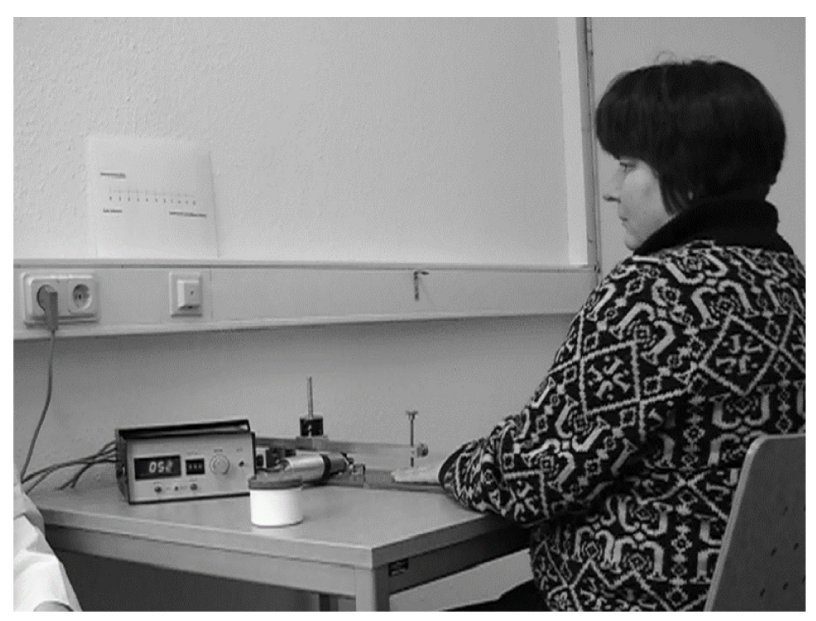

Figure 2 Still picture of the pressure pain application video. Notes: Pressure pain is applied to the model's left ring finger. Only the experimenter's hands were visible during the entire video. effect of the ointment on the pain ratings. The model's ratings for the hand with ointment were identical to those without the ointment, ie, ranging from 2 to 3 . The videos were identical in facial expressions, body posture, and tone of voice.

\section{Materials}

Pain was induced by a stationary pressure pain algometer, which delivered constant pressure over a fixed period. The algometer consisted of a lever with a weight, and a plunger with a surface area of $3 \mathrm{~mm}^{2}$ was located at the end. An electric motor lowered the lever with the plunger onto the finger when a button was pressed, and the lever rose automatically after a predetermined period. As a safety measure, the procedure could be stopped immediately by pushing a button. A weight of $300 \mathrm{~g}$ set at $8 \mathrm{~cm}$ was used, resulting in a total pressure of $0.92 \mathrm{MPa}$, which was applied for 60 seconds.

A standard hypoallergenic ointment served as the nocebo. It contained the following ingredients: aqua, caprylic/capric triglyceride, glycerin, pentylene glycol, Cocos nucifera, hydrogenated lecithin, Vitellaria paradoxa, hydroxyethylcellulose, squalane, sodium carbomer, xanthan gum, carbomer, and ceramide 3 . It was presented in a neutral container, similar to those used by dispensing chemists, and contained no perfume. The ointment itself had no pharmacological influence on pain perception.

\section{Measures}

Since the pain measures and empathy were directly related to our experimental design, we decided to collect these measures after the application of pain to avoid any speculations about the aim of our study. In order to reduce the time for the participants in our laboratory, they were sent the other questionnaires (not concerning the pain measures or empathy) to fill in at home prior to the appointment.

The trait pain catastrophizing was measured with the German version of the PCS, which consisted of 13 items answered on a five-point scale ranging from 0 ("not at all") to 4 ("all the time"). ${ }^{17,18}$ A sum score ( $\min =0, \max =52$ ) and scores for each of the three subscales were calculated. The subscale "Rumination" ( $\min =0, \max =16$ ) described the inability to stop thoughts concerning pain. The items of the subscale "Magnification" ( $\min =0, \max =12$ ) reflected the tendency to exaggerate the threat value of pain stimuli. "Helplessness" ( $\min =0, \max =24)$ described the inability to deal with pain.

Unspecific somatic complaints were measured with the German Symptom Inventory (Beschwerdeliste, BL-R). ${ }^{19}$ The participants indicated the degree to which they suffered from 
each somatic symptom (eg, constipation, insomnia, shortness of breath, or lack of energy) on a four-point scale ranging from 0 ("not at all") to 3 ("strongly"). There were two parallel versions: each consisted of 24 items ( $\min =0, \max =72$ for each version). In order to improve reliability, we used both versions (ie, 48 items) and averaged the values.

To measure hypochondriacal concerns, the German version of the WI was used. It consisted of 14 items that the participant indicated as present or not $(\min =0, \max =14) .{ }^{20}$

The German version of the IRI (Saarbrücker Persönlichkeitsfragebogen) was used to assess trait empathy. ${ }^{21,22}$ The questionnaire consisted of 16 items answered on a five-point scale ranging from 1 ("never") to 5 ("always") with the subscale "Empathic Concern", "Perspective-taking", "Fantasy", and "Personal Distress" ( $\min =4, \max =20$, for each scale). The global score $(\min =12, \max =60)$ was calculated by summing the scales "Empathic Concern", "Perspective-taking", and "Fantasy". 23

As control measures, depression and anxiety were assessed with the German version of the HADS-D. ${ }^{24}$ The HADS-D is a 14-item self-report scale designed to measure depression and anxiety symptoms in the previous week. The items were answered on a four-point scale with item-specific response categories $(\min =0, \max =21$ for each scale).

In addition, participants were asked to rate the credibility of the cover story on a scale ranging from 0 ("not credible at all") to 10 ("completely credible").

\section{Hypotheses}

The following hypotheses were tested:

1. An interaction exists between condition and ointment application for the pain ratings.

2. In the $\mathrm{NC}$, pain ratings are higher with ointment than without ointment.

3. The pain ratings with ointment are higher in the $\mathrm{NC}$ than in the $\mathrm{CC}$.

4. In the $\mathrm{NC}$, positive correlations exist between the nocebo response and empathy, pain catastrophizing, unspecific somatic complaints, and hypochondriacal concerns.

\section{Data analysis}

Differences regarding age, depression, anxiety, pain catastrophizing, unspecific somatic complaints, hypochondriacal concerns, and the credibility ratings between the participants in the two conditions were compared with independent $t$-tests.

Prior tests showed that the assumptions for a $2 \times 2$ repeated measures analysis of variance (ANOVA) were fulfilled: the data were normally distributed (Kolmogorov-Smirnov test) and variances were homogeneous (Levene's test). In order to test the interaction hypothesis for the nocebo effect, the mean pain intensity score for each hand was calculated, and a $2 \times 2$ repeated measures ANOVA with between-subject factor condition $(\mathrm{NC} / \mathrm{CC})$ and within-subject factor application of ointment (yes/no) was computed. Planned contrasts were calculated using independent or paired $t$-tests as appropriate. As measures of effect sizes, $\eta^{2}$ and Cohen's $d$ were calculated.

The nocebo response for each individual participant was determined by the difference between the mean pain intensity ratings with and without ointment for that participant. Higher values indicated a stronger nocebo response.

Pearson correlations were computed for each condition for the nocebo response with empathy, pain catastrophizing, unspecific somatic complaints, and hypochondriacal concerns. The level of significance was set at $P<0.05$. All analyses were carried out with STATISTICA for Windows software, version 10 (StatSoft Inc., Tulsa, OK, USA).

\section{Results}

There were no differences in depression, anxiety, empathy, pain catastrophizing, the score on the complaints list, and hypochondriacal concerns between the conditions (Table 1). The $t$-test of the credibility ratings for the information concerning the ointment revealed no differences between the two conditions $(t[92]=0.05, P=0.96)$.

The $2 \times 2$ repeated measures ANOVA revealed a significant effect of condition $\left(F[1,95]=4.76, P=0.03, \eta^{2}=0.04\right)$ as well as a significant effect of ointment application $\left(F[1,95]=26.62, P=0.000001, \eta^{2}=0.04\right)$ on the pain ratings. According to Cohen, ${ }^{25}$ these were small effects. There was no interaction between condition $\mathrm{x}$ ointment $(F[1,95]=0.68$, $P=0.41]$. The paired $t$-test identified higher pain intensity with as compared to without ointment within the $\mathrm{NC}(t[46]$ $=3.57, P=0.0008, d=0.38$ ). According to Cohen, the effect was of approximately medium size. ${ }^{25}$ The independent $t$-test identified higher pain intensity with ointment in the $\mathrm{NC}$ than in the $\mathrm{CC}(t[95]=-2.18, P=0.03, d=0.44)$. The effect was of a medium size. Exploratory analyses identified higher pain intensities with as compared to without ointment within the $\mathrm{CC}(t[49]=3.82, P=0.0004, d=0.38)$ and no differences in pain ratings without ointment between $\mathrm{CC}$ and $\mathrm{NC}(t[95]$ $=-1.85, P=0.07, d=0.37$ ) (Figure 3 and Table 2).

For the $\mathrm{NC}$, none of the expected correlations were observed (Table 3 ). In the $\mathrm{CC}$, exploratory analyses revealed that the nocebo response was positively correlated with the 


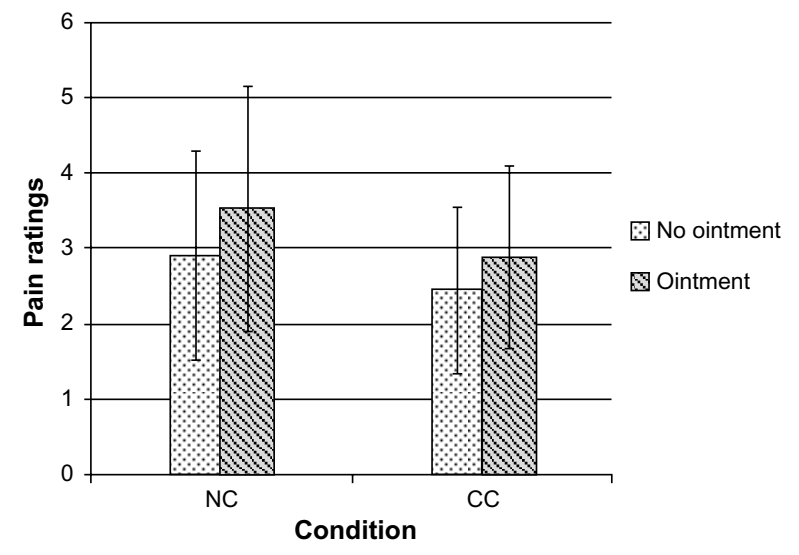

Figure 3 Pain as a function of condition and ointment application.

Note: Numerical pain ratings on a sale from 0 , "no pain" to 10 , "worst imaginable pain". Error bars represent standard deviations.

Abbreviations: NC, nocebo condition; CC, control condition.

sum score of the symptom inventory $(r=0.34, P<0.05)$ and the sum score of the WI $(r=0.32, P<0.05)$.

\section{Discussion}

We examined whether a nocebo hyperalgesia to pressure pain could be induced by social observational learning in a female general population sample. There was no interaction between condition and ointment application. However, main effects for condition and ointment application on the pain ratings were observed. Planned comparisons revealed that, within the NC, participants reported more pain with as compared to without ointment, so a nocebo effect for the $\mathrm{NC}$ was observed. Unexpectedly, pain ratings in the $\mathrm{CC}$ with ointment were higher than without, so a pain increase after ointment application was observed in the $\mathrm{CC}$ too. Due to the unexpected pain increase with ointment in the $\mathrm{CC}$, no interaction was observed. The pain ratings with ointment were higher in the $\mathrm{NC}$ than in the $\mathrm{CC}$, indicating that the observation of the model had an additional effect on pain perception. Contrary to our hypothesis, in the NC, no correlation between the nocebo response and empathy, pain catastrophizing, hypochondriacal concerns, or the amount of bodily symptoms was observed. In contrast, in the CC,

Table 2 Pain ratings on the NRS 0-10 with and without ointment and nocebo responses for the nocebo and control conditions

\begin{tabular}{llllll}
\hline Condition & NC & & & CC & \\
\cline { 2 - 3 } \cline { 5 - 6 } & M & SD & & M & SD \\
\hline Without ointment & 2.9 & 1.4 & & 2.4 & 1.1 \\
With ointment & 3.5 & 1.6 & & 2.9 & 1.2 \\
Nocebo response & 0.6 & 1.2 & & 0.4 & 0.8 \\
\hline
\end{tabular}

Abbreviations: NRS, numerical rating scale; NC: nocebo condition; CC, control condition; M, mean; SD, standard deviation.
Table 3 Correlations between the nocebo response and IRI, PCS, BL-R, and WI in the nocebo and control conditions

\begin{tabular}{lll}
\hline Questionnaires & $\mathbf{N C}$ & $\mathbf{C C}$ \\
& $\mathbf{N}=\mathbf{4 5}$ & $\mathbf{N}=\mathbf{4 8}$ \\
& $\mathbf{r}$ & $\mathbf{r}$ \\
\hline IRI & 0.16 & 0.20 \\
Empathic Concern & 0.11 & 0.08 \\
Fantasy & 0.17 & 0.07 \\
Personal Distress & -0.16 & -0.10 \\
Perspective-taking & 0.09 & 0.19 \\
PCS & -0.03 & 0.00 \\
Rumination & -0.02 & -0.18 \\
Magnification & 0.03 & 0.05 \\
Helplessness & -0.07 & 0.17 \\
BL-R & -0.14 & $0.34^{*}$ \\
WI & -0.00 & $0.32^{*}$ \\
\hline
\end{tabular}

Note: $* P<0.05$.

Abbreviations: NC, nocebo condition; CC, control condition; IRI, Interpersonal Reactivity Index; PCS, Pain Catastrophizing Scale; BL-R, Symptom Inventory (Beschwerdeliste); WI, Whiteley Index.

we found that the higher a person's level of hypochondriacal concerns was and the more bodily symptoms she reported, the higher was the nocebo response.

The social observation induced elevated pain ratings to pressure pain, since participants in the NC who watched a model demonstrating higher pain ratings after the application of an ointment rated pressure pain with ointment as more painful than the participants in the $\mathrm{CC}$ who watched a model demonstrating no change in pain perception after the application of an ointment. By watching a video in which pain was demonstrated by a model, the participants might have built up the expectation of pain when an ointment is applied. The expectation of pain might have led to anxiety, which facilitates pain perception. ${ }^{26}$ According to Benedetti et a ${ }^{26}$ and Colloca and Benedetti, ${ }^{27}$ anxiety is an important mechanism in nocebo hyperalgesia. Hyperalgesia is known to occur when anticipatory anxiety regarding pain itself is experienced, and also if attention is directed toward the pain. ${ }^{5,26,27}$ These processes may have had an effect on pain perception in the NC.

In addition, ointment application induced higher pain ratings, since participants rated pain with the ointment as more painful than without in the $\mathrm{CC}$. We can rule out the possibility that any active ingredient of the ointment altered pain perception, since the ointment did not contain any active substances. In addition, in our last study, no such effects were observed. ${ }^{4}$ Due to the common practice of using ointments for pain relief, the ointment could have triggered the expectation of pain relief despite the information from the video. It is unclear why a pain increase emerged. Perhaps the psychosocial context, which has been shown to strongly influence 
nocebo effects, triggered the expectation of an increase in pain. ${ }^{26,27}$ The experimenters wore white medical coats and gloves; medical supplies were in the room and it smelled of disinfectant spray. This may have led to the expectation that something painful was imminent that required the prior application of an ointment. Therefore, the participants might have built up the expectation of pain when the ointment was applied.

Due to the observation of a video model, which demonstrated higher pain after the application of an ointment, pain ratings in the $\mathrm{NC}$ with ointment were higher than without ointment and higher than with ointment in the $\mathrm{CC}$. The pain increase due to the ointment application in the CC explained why no nocebo (interaction) effect was observed.

The described results were compatible with the results of our former study. ${ }^{4}$ In the verbal CC of that study, we provided explicit verbal information that the ointment would have no effect on pain experience and, consequently, observed no nocebo response. In the presence of less definite information, as in our present study, the ointment application might have activated the expectation of pain, as argued earlier.

Contrary to our expectation and the results of our previous study, we found no correlation between pain catastrophizing and nocebo response in the $\mathrm{NC} .{ }^{4}$ Neither did we find an association between individual nocebo response and unspecific somatic complaints or hypochondriasis. The absence of such effects is in line with earlier studies, in which attempts to find variables predicting the placebo response failed to produce strong or consistent results. ${ }^{28,29}$ For the placebo response, it was concluded that the situation should be taken into account and the interaction of situational variables and personality traits should be investigated. ${ }^{28,29}$ The same may be concluded for the nocebo response.

Unexpectedly, in the $\mathrm{CC}$, the symptom inventory and the nocebo response were positively associated (moderate effect size): the more somatic symptoms a person reported, in general, the more likely she was to report higher pain after the application of the ointment. This result may have reached significance by chance and should be replicated. If future research confirms these associations, a reason for this could lie in a top-down driven symptom perception, a process especially prominent in people with a tendency to report many negative somatosensory events. ${ }^{30}$ Top-down driven processes may contribute to turning bodily sensations into symptoms by mobilizing schematic information resulting from the person's learning history. The schema influences the allocation of attention and the way sensations are interpreted or appraised. ${ }^{7,9,30}$ In the $\mathrm{CC}$, people with many unexplained symptoms and a more top-down driven processing style may have ignored the diverging information that the ointment does not change pain perception and relied more on their schema "ointment application means pain". As a result, their pain perception may have increased when the ointment was applied. In the presence of the information that the ointment increases pain perception (NC), the individual tendency for such interpretations had no influence, and an increase in pain was experienced irrespective of the level of symptom reporting.

In a similar vein, a positive correlation between hypochondriacal concerns and the nocebo response was observed in the CC. The same mechanisms explained earlier can be applied for this result. This was in line with the findings that health-anxious people focused on their own negative reactions to pain, failed to make use of positive information adequately, and reported more catastrophic thoughts about the meaning and implications of pain. ${ }^{31}$ One possible explanation could be that the more health anxious a person was, the less she made use of the positive information that the ointment does not change the pain experience.

No correlation between trait empathy and nocebo response was found. This reproduced the negative result obtained in our previous study. ${ }^{4}$ In that study, we could not exclude that the negative result was due to the limited variability of the - rather high - empathy scores in our sample. In the present study, a community sample with more variability (empathy scale standard deviation $[\mathrm{SD}]=5.2$ as opposed to 3.3 in our last study) was tested, and still, no correlation was found. In the study by Swider and Babel, the subscales "Empathic Concern" and "Personal Distress" predicted the nocebo response. ${ }^{3}$ In a recent study comparing a face-to-face versus a prerecorded observation of placebo analgesia, Hunter et al found no correlation between empathic concern and placebo analgesia in the video replay group, but they did observe a correlation in the live observation group. ${ }^{32}$ Although this has not been investigated for nocebo hyperalgesia, the video presentation of the model could possibly explain the lack of correlation between trait empathy and nocebo response.

The study had several methodological strengths. A professional, medical environment was created to strengthen the trust of the participants in the investigation. The credibility ratings of the cover story were high and there were no differences between the conditions, indicating that the instruction was accepted by all participants. After the experiment, we asked the participants to guess the purpose of the study. In order to classify the responses, four independent raters judged the participants' answers to see whether they might have 
discovered the real aim of our study. Only three participants speculated about placebo/nocebo effects as the topic of research (they were not excluded from the analysis).

Experimenter expectations may exert an influence on the results in placebo studies, and it is reasonable to think that the same may be true for nocebo studies. ${ }^{33}$ In order to forestall such effects, the experimenters in the current study were blind with regard to the participants' assigned condition. In addition, they were trained with the support of a video to conduct the experiment in a highly standardized manner. In summary, we did not detect any factor endangering internal validity.

Expectations are crucial to the nocebo response. ${ }^{26,27}$ We did not ask the participants about their expectations regarding the ointment application and, therefore, can only indirectly infer that such expectations were induced. Our results suggested that we induced the expectation of increased pain in both the conditions. In future studies, the expectations should be directly assessed.

Our study was not without limitations. One limitation related to the sample tested: all participants were women and they observed a female model; thus, the results were limited to observational learning in women with female models. Since we investigated acute, experimentally induced pain, it remains an open question whether our results apply to naturally occurring or chronic pain. We did not control for the effect of anxiety, which might have facilitated a nocebo response. ${ }^{26,27}$ In our last study, however, we did not find an association between nocebo response and state or trait anxiety. ${ }^{4}$

\section{Conclusion}

As hypothesized, the observation of a model demonstrating pain led to an increase in the pain experience, so, again, a socially induced nocebo effect was observed. In addition, the application of an ointment led to higher pain ratings, possibly by way of invoking pain schemas. It appears that, in the absence of definite information, health- and body-related cognitive styles influence these nocebo responses. If further studies confirm our results that even the observation of pain in general or the unexplained administration of an intervention can lead to hyperalgesia, this may have implications for clinical trials concerned with pain and its assessment. In addition, these considerations could be applied to minimize the unnecessary expectation-induced pain in medical procedures.

\section{Acknowledgment}

The authors gratefully acknowledge the help of Lisa Wedekind, Katharina Haas, and Viola Ranft who helped with the data collection. The authors also acknowledge support by the German Research Foundation and the Open Access Publication Funds of the University of Göttingen.

\section{Author contributions}

All authors listed participated in the research and article preparation. Each author made substantial contributions to the conception and design of the study as well as the analysis and interpretation of the data and drafting and critically revising the paper. All authors read and approved the final version of the article.

\section{Disclosure}

The authors report no conflicts of interest in this work.

\section{References}

1. Colloca L, Sigaudo M, Benedetti F. The role of learning in nocebo and placebo effects. Pain. 2008;136(1-2):211-218.

2. Van Laarhoven AIM, Vogelaar ML, Wilder-Smith OH, et al. Induction of nocebo and placebo effects on itch and pain by verbal suggestions. Pain. 2011;152(7):1486-1494.

3. Świder K, Babel P. The effect of the sex of a model on nocebo hyperalgesia induced by social observational learning. Pain. 2013;154(8):13121317. doi:10.1016/j.pain.2013.04.001.

4. Vögtle E, Barke A, Kröner-Herwig B. Nocebo hyperalgesia induced by social observational learning. Pain. 2013;154(8):1427-1433. doi:10.1016/j.pain.2013.04.041.

5. Benedetti F. Placebo and the new physiology of the doctor-patient relationship. Physiol Rev. 2013;93(3):1207-1246. doi:10.1152/ physrev.00043.2012.

6. Crombez G, Wiech K. You may (not always) experience what you expect: in search for the limits of the placebo and nocebo effect. Pain. 2011;152(7):1449-1450. doi:10.1016/j.pain.2011.02.028.

7. Brown RJ. Psychological mechanisms of medically unexplained symptoms: an integrative conceptual model. Psychol Bull. 2004;130(5):793-812. doi:10.1037/0033-2909.130.5.793.

8. Bayer TL, Coverdale JH, Chiang E, Bangs M. The role of prior pain experience and expectancy in psychologically and physically induced pain. Pain. 1998;74(2-3):327-331.

9. Rief W, Broadbent E. Explaining medically unexplained symptomsmodels and mechanisms. Clin Psychol Rev. 2007;27(7):821-841. doi:10.1016/j.cpr.2007.07.005.

10. Watt MC, Stewart SH. Anxiety sensitivity mediates the relationships between childhood learning experiences and elevated hypochondriacal concerns in young adulthood. $J$ Psychosom Res. 2000;49(2):107-118.

11. Slavicek G, Forsdahl G. Ethics and regulatory aspects in medical research. Int J Stomatol Occlusion Med. 2009;2(1):45-49.

12. Racine M, Tousignant-Laflamme Y, Kloda LA, Dion D, Dupuis G, Choinière $\mathrm{M}$. A systematic literature review of 10 years of research on sex/gender and experimental pain perception - Part 1: are there really differences between women and men? Pain. 2012;153(3):602-618. doi:10.1016/j.pain.2011.11.025.

13. Racine M, Tousignant-Laflamme Y, Kloda LA, Dion D, Dupuis G, Choinière M. A systematic literature review of 10 years of research on sex/gender and pain perception - Part 2: do biopsychosocial factors alter pain sensitivity differently in women and men? Pain. 2012;153(3): 619-635. doi:10.1016/j.pain.2011.11.026.

14. Kállai I, Barke A, Voss U. The effects of experimenter characteristics on pain reports in women and men. Pain. 2004;112(1):142-147. 
15. Lorber W, Mazzoni G, Kirsch I. Illness by suggestion: expectancy, modeling, and gender in the production of psychosomatic symptoms. Ann Behav Med. 2007;33(1):112-116.

16. Faul F, Erdfelder E, Lang AG, Buchner A. G* Power 3: a flexible statistical power analysis program for the social, behavioral, and biomedical sciences. Behav Res Methods. 2007;39(2):175-191.

17. Sullivan MJL, Bishop SR, Pivik J. The Pain Catastrophizing Scale: development and validation. Psychol Assess. 1995;7(4):524-532.

18. Meyer K, Sprott H, Mannion AF. Cross-cultural adaptation, reliability, and validity of the German version of the Pain Catastrophizing Scale. J Psychosom Res. 2008;64(5):469-478.

19. Zerssen D, Koeller DM. Beschwerden-Liste. [German Symptom Inventory]. 1st ed. Weinheim: Beltz Test GmbH; 1976. German.

20. Hiller W, Rief W. Internationale Skalen für Hypochondrie. [International scale for hypochondriasis]. 1st ed. Bern: Hans Huber; 2004. German.

21. Davis MH. A multidimensional approach to individual differences in empathy. JSAS Catalog of selected Documents in Psychology. 1980;10:85.

22. Paulus C. Saarbrücker Persönlichkeits-Fragebogen. [Interpersonal Reactivity Index]. 2006. Available from: http://search.ebscohost.com/ login.aspx?direct $=$ true $\& d b=p d x \& A N=P T 9005661 \&$ site=ehost-live. Translated from English to German.

23. Paulus $\mathrm{C}$. The possibility of a general empathy score in the German version of the IRI Ist die Bildung eines Empathiescores in der deutschen Fassung des IRI sinnvoll. [The possibility of a general empathy score in the German version of the IRI?]. Available from: http:// scidok.sulb.uni-saarland.de/volltexte/2012/4889/. German. Accessed July 5,2013
24. Herrmann-Lingen C, Buss U, Snaith RP. HADS-D Hospital Anxiety and Depression Scale. 3rd ed. Bern: Hans huber; 2011.

25. Cohen J. A power primer. Psychol Bull. 1992;112:155-159.

26. Benedetti F, Lanotte M, Lopiano L, Colloca L. When words are painful: unraveling the mechanisms of the nocebo effect. Neuroscience. 2007;147:260-271.

27. Colloca L, Benedetti F. Nocebo hyperalgesia: how anxiety is turned into pain. Curr Opin Anesthesiol. 2007;20(5):435

28. Jakšić N, Aukst-Margetić B, Jakovljević M. Does personality play a relevant role in the placebo effect. Psychiatr Danub. 2013;25(1): $17-23$.

29. Geers AL, Kosbab K, Helfer SG, Weiland PE, Wellman JA. Further evidence for individual differences in placebo responding: an interactionist perspective. J Psychosom Res. 2007;62(5):563-570.

30. Bogaerts K, Van Eylen L, Li W, et al. Distorted symptom perception in patients with medically unexplained symptoms. J Abnorm Psychol. 2010;119(1):226-234. doi:10.1037/a0017780.

31. Hadjistavropoulos HD, Craig KD, Hadjistavropoulos T. Cognitive and behavioral responses to illness information: the role of health anxiety. Behav Res Ther. 1998;36(2):149-164.

32. Hunter T, Siess F, Colloca L. Socially induced placebo analgesia: a comparison of a pre-recorded versus live face-to-face observation: social learning, empathy and placebo analgesia. EJP. 2014;18(7):914922. doi:10.1002/j.1532-2149.2013.00436.x.

33. Walach H, Schimdt S, Bihr Y-M, Wiesch S. The effects of a caffeine placebo and experimenter expectation on blood pressure, heart rate, well-being, and cognitive performance. Eur Psychol. 2001;6(1):15.
Journal of Pain Research

\section{Publish your work in this journal}

The Journal of Pain Research is an international, peer-reviewed, open access, online journal that welcomes laboratory and clinical findings in the fields of pain research and the prevention and management of pain. Original research, reviews, symposium reports, hypothesis formation and commentaries are all considered for publication.

\section{Dovepress}

The manuscript management system is completely online and includes a very quick and fair peer-review system, which is all easy to use. Visit http://www.dovepress.com/testimonials.php to read real quotes from published authors. 